\title{
UPPER BOUND FOR DISTORTION OF CAPACITY UNDER CONFORMAL MAPPING
}

\author{
ROBERT E. THURMAN
}

\begin{abstract}
For a finitely-connected domain $\Omega$ containing $\infty$, with boundary $\Gamma$, the logarithmic capacity $d(\Gamma)$ is invariant under normalized conformal maps of $\Omega$. But the capacity of a subset $A \subset \Gamma$ will likely be distorted by such a map. Duren and Schiffer showed that the sharp lower bound for the distortion of the capacity of such a set is the so-called "Robin capacity" of the set $A$. We present here the sharp upper bound for the distortion, in terms of conformal invariants of $\Omega$ : the harmonic measures of the boundary components of $\Omega$ and the periods of their harmonic conjugates (the Riemann matrix), and the capacity of $\Gamma$. In particular, the upper bound depends only on knowing which components of $\Gamma$ contain parts of $A$, not on the specific distribution of $A$. An extremal configuration is described explicitly for a special case.
\end{abstract}

\section{INTRODUCTION}

It is well known that logarithmic capacity (transfinite diameter) remains invariant under normalized conformal mappings. Specifically, let $\Omega \subset \widehat{\mathbf{C}}$ be a finitely-connected domain containing infinity, and let $\Gamma$ be its boundary. Then the logarithmic capacity $d(\Gamma)$ remains unchanged under conformal maps $f$ of $\Omega$ which are normalized so that $f(\infty)=\infty$, and $f^{\prime}(\infty)=1$ :

$$
f(\zeta)=\zeta+b_{0}+\frac{b_{1}}{\zeta}+\frac{b_{2}}{\zeta^{2}}+\ldots
$$

near infinity.

However, the capacity of a subset $A \subset \Gamma$ will probably be distorted under such a map. For example, if $\Omega$ is the outside of the unit disk, and $A$ is the top half of the unit circle, then it is known that $d(\Gamma)=1$ and $d(A)=\sin \left(\frac{\pi}{4}\right)=\frac{1}{\sqrt{2}}$. But under the normalized Joukowski map $f(\zeta)=\zeta+1 / \zeta$, the set $A$ gets taken to one side of the line segment $-2 \leq x \leq 2$, which is the full boundary of the image, so that

$$
d(f(A))=d(f(\Gamma))=d(\Gamma)=1 .
$$

Thus the capacity of $A$ is increased to the capacity of the full boundary under this conformal mapping.

Received by the editors November 23, 1993.

1991 Mathematics Subject Classification. Primary 30C70, 30C85; Secondary 30C20.

Key words and phrases. Conformal mapping, logarithmic capacity, extremal problems, variational methods, theta functions, Green's function, harmonic measure, multiply-connected domains. 
Duren and Schiffer [3] have given the sharp lower bound for distortion of the capacity of any subset:

$$
d(f(A)) \geq \delta(A)
$$

where $\delta(A)$ denotes the "Robin capacity" of the set $A$. We present here the sharp upper bound. Since $f(A) \subseteq f(\Gamma)$, we trivially have

$$
d(f(A)) \leq d(f(\Gamma))=d(\Gamma) .
$$

And whenever each component of $\Gamma$ contains points of $A$, even a single point, we show that it is possible to achieve equality, using a method suggested by the above example.

If some component of $\Gamma$ contains no part of $A$, we will see that the maximal distortion is strictly less than the capacity of the full boundary:

$$
d(f(A)) \leq d(\Gamma) e^{-C}, \quad C>0 .
$$

The constant $C$ giving the sharp bound is calculated in terms of standard conformal invariants of the domain $\Omega$ : the harmonic measures of the boundary components and the periods of their harmonic conjugates (the Riemann matrix), and the capacity of $\Gamma$. In particular, the maximal capacity depends only on knowing which components of $\Gamma$ contain parts of $A$, not on the actual distribution.

We also characterize extremal domains $F(\Omega)$ in terms of Green's function $\widehat{g}(z ; \infty)$ for the larger domain bounded only by $F(A)$ : The capacity $d(F(A))$ is maximal if and only if $\hat{g}$ is constant on each component of $F(\Gamma) \backslash F(A)$.

The strategy of the paper is to first consider the special case where $A$ is the union of some collection of full components of $\Gamma$. A variational method provides enough information about extremal configurations to compute the maximum distortion. We then reduce the general case to the special case. In the final section, we describe an extremal domain for the case where $A$ is a single, full component of $\Gamma$. We conclude with an example where $\Omega$ is the exterior of iwo circles, one of which is the unit circle, and the other which is $A$. The maximal capacity is computed explicitly in terms of the radius and center of $A$, and is the same if $A$ is any nonempty subset of that circle.

We will be using the potential-theoretic definition for the capacity of a compact set $K$ : If $D$ is the component of $\widehat{\mathbf{C}} \backslash K$ containing infinity, and $g(z ; \infty)$ is Green's function for $D$ with singularity at infinity, then $d(K)=e^{-\gamma}$, where $\gamma$ is Robin's constant, given by

$$
\gamma=\lim _{z \rightarrow \infty}\{g(z ; \infty)-\log |z|\} .
$$

To fix the notation, we will refer to the family of normalized conformal maps of $\Omega$ as $\mathscr{F}$, and say that $f$ is admissible if $f \in \mathscr{F}$. Green's function for the domain $f(\Omega)$, where $f$ is admissible, will be denoted $\tilde{g}(z)=\tilde{g}(z ; \infty)$. To compute $d(f(A))$, we will need Green's function for the domain containing $f(\Omega)$ and bounded only by $f(A)$. We will let $\widehat{\Omega}$ denote this domain, and $\widehat{g}(z)=\widehat{g}(z ; \infty)$ its Green's function. The dependence of $\widetilde{g}, \widehat{g}$, and $\widehat{\Omega}$ on the particular admissible map $f$ should be clear from the context. 


\section{A SPECIAL CASE}

Let $\Gamma$ consist of the disjoint Jordan curves $\Gamma_{1}, \ldots, \Gamma_{m}$. We first consider the special case where $A$ is a union of boundary components:

$$
A=\Gamma_{1} \cup \cdots \cup \Gamma_{k}, \quad k<m .
$$

The main result is stated in the following theorem.

Theorem 1. Let $\Omega$ be a domain containing $\infty$ and bounded by the Jordan curves $\Gamma_{1}, \ldots, \Gamma_{m}$. For $k<m$, let $A=\bigcup_{i=1, \ldots, k} \Gamma_{i}$ and $B=\bigcup_{i=k+1, \ldots, m} \Gamma_{i}$. Let $\omega_{i}(\zeta)$ be the harmonic measure of $\Gamma_{i}$ with respect to $\Omega$, and set $v_{i}=\omega_{i+k}(\infty)$ for $i=1, \ldots, m-k$. Let $p_{i j}$ be the period of the harmonic conjugate of $\omega_{i+k}$ about $\Gamma_{j+k}$ for $i, j=1, \ldots, m-k$. Setting $\mathbf{v}=\left(v_{1}, \ldots, v_{m-k}\right)$ and $\mathbf{P}=\left(p_{i j}\right)$, define $\mathbf{c}=\left(c_{1}, \ldots, c_{m-k}\right)$ by

$$
\mathbf{c}=\mathbf{P}^{-1} \mathbf{v}
$$

Then if $f$ is any normalized conformal map of $\Omega$,

$$
d(f(A)) \leq d(\Gamma) \exp \left\{-\sum_{i=1}^{m-k} c_{i} v_{i}\right\} .
$$

The inequality is sharp. Extremal domains $F(\Omega)$ exist and are characterized by the property that each of the $m-k$ components $\widetilde{B}_{i}$ of $F(B)$ is a union of analytic arcs, and Green's function $\widehat{g}(z ; \infty)$ of the domain $\widehat{\Omega} \supset F(\Omega)$ bounded only by $F(A)$ is constant on each $\widetilde{B}_{i}$. In other words, $\widehat{g}(z ; \infty)=a_{i}$ on $\widetilde{B}_{i}$ for each $i=1, \ldots, m-k$ and some constants $a_{i}>0$, if and only if $d(F(A)) \geq$ $d(f(A))$ for all normalized conformal maps $f$ of $\Omega$. In this extremal case, $a_{i}=c_{i}$ as defined in (2).

Note that $\mathbf{P}$ is the $(m-k) \times(m-k)$ submatrix, corresponding to $\boldsymbol{B}$, of the standard Riemann matrix for $\Omega$.

The proof is in several steps. We first prove the existence of extremal maps. Then we apply a boundary variation to an extremal domain. This leads to the result that $\widehat{g}$ must be constant on each $\widetilde{B}_{i}$. We are then able to compute the constants and calculate the maximal capacity. Finally, we show that these calculations do not depend on the extremal character of the domain, but only on the fact that $\widehat{g}$ is constant on each $\widetilde{B}_{i}$.

Proof of theorem. We begin by using a compactness argument to infer the existence of extremal maps. We may assume that our admissible maps of the form (1) further satisfy $b_{0}=0$, since subtracting a constant will not affect the capacity. The advantage here is that the resulting family $\mathscr{T}_{0}$ is compact and normal (see Wen $\left[9\right.$, p. 105], for example). Let a sequence of functions $\left\{f_{n}\right\} \subseteq \mathscr{F}$ have the property that

$$
\lim _{n \rightarrow \infty} d\left(f_{n}(A)\right)=\sup _{f \in \mathscr{F}_{0}} d(f(A)) .
$$

By normality and compactness, we may assume that $\left\{f_{n}\right\}$ converges locally uniformly in $\Omega$ to a function $F \in \mathscr{F}$. We need to show

$$
\lim _{n \rightarrow \infty} d\left(f_{n}(A)\right) \leq d(F(A))
$$


To this end, let $\widehat{\Omega} \supseteq F(\Omega)$ be the domain bounded only by $F(A)$, with associated Green's function $\widehat{g}(z ; \infty)$. Let $\widehat{\Omega}_{n} \supseteq f_{n}(\Omega)$ be the domains bounded only by $f_{n}(A)$, with associated Green's functions $\widehat{g}_{n}(z ; \infty)$. By a general form of the Carathéodory convergence theorem (see Goluzin [4, Chapter 5, $\S 5]$ ), the domains $f_{n}(\Omega)$ converge in the sense of Carathéodory to $F(\Omega)$. In particular, the domains $\widehat{\Omega}_{n}$ converge to $\widehat{\Omega}$. To show (4), let $\epsilon>0$ be given, and consider the following closed subset of $\widehat{\Omega}$ :

$$
K=\{z: \widehat{g}(z) \geq \epsilon\} \cup\{\infty\} .
$$

Then $\widehat{g}(z) \equiv \epsilon$ on the level curve $K_{\epsilon}=\partial K$, which surrounds $F(A)$. Now since $\widehat{\Omega}_{n} \rightarrow \widehat{\Omega}$, we have that $K \subset \widehat{\Omega}_{n}$ for large enough $n$. For all such $n$, then, $\widehat{g}_{n}$ is defined on $K \backslash\{\infty\}$, and of course $\widehat{g}_{n}>0$ there. But then the functions $\widehat{g}_{n}-\widehat{g}$ are harmonic in all of $K$, and satisfy $\widehat{g}_{n}-\widehat{g}>-\epsilon$ on $K_{\epsilon}$. Thus $\widehat{g}_{n}-\widehat{g}>-\epsilon$ in all of $K$, so that

$$
-\epsilon<\lim _{z \rightarrow \infty}\left\{\left(\widehat{g}_{n}(z)-\log |z|\right)-(\widehat{g}(z)-\log |z|)\right\}=\gamma_{n}-\gamma .
$$

Therefore,

$$
d\left(f_{n}(A)\right)=e^{-\gamma_{n}}<e^{-\gamma} e^{\epsilon}=d(F(A)) e^{\epsilon}, \quad n \geq N .
$$

Since $\epsilon>0$ is arbitrary, this proves (4). It follows that $F$ is extremal, or that $d(F(A)) \geq d(f(A))$ for all admissible maps of $\Omega$.

The next step is to apply a boundary variation to obtain information about an extremal domain. Let $\widetilde{\Omega}=F(\Omega)$ where $F$ is an extremal function. Then the definition of the boundary sets $\widetilde{A}, \widetilde{B}, \widetilde{\Gamma}$, and $\widetilde{\Gamma}_{i}$ is clear, and $d(\widetilde{A}) \geq d(f(\widetilde{A}))$ for all admissible maps $f$. For each fixed point $z_{0}$ in $\widetilde{B}$, there is a family of functions

$$
V_{\rho}(z)=z+\frac{a \rho^{2}}{z-z_{0}}+O\left(\rho^{3}\right)
$$

analytic and univalent in the complement of some small connected subset of $\widetilde{B}$ containing $z_{0}$ (see Duren $[2, \S 10.3]$ ). In particular, each of the boundary variations $V_{\rho}(z)$ acts also as an interior variation on the domain $\widehat{\Omega}$ bounded only by $\widetilde{A}$. Let $A^{*}=V_{\rho}(A)$. A known variational formula [8] gives that

$$
d\left(A^{*}\right)=d(\widetilde{A})\left[1-\operatorname{Re}\left\{a \rho^{2} \hat{p}^{\prime}\left(z_{0}\right)^{2}\right\}+O\left(\rho^{3}\right)\right],
$$

where $\widehat{p}(z)$ is the analytic completion of Green's function $\widehat{g}(z)$ of $\widehat{\Omega}$. But $V_{\rho}$ is also an admissible map of $\widetilde{\Omega}$. Thus $d(\widetilde{A}) \geq d\left(A^{*}\right)$, and so

$$
\operatorname{Re}\left\{-a \rho^{2} \hat{p}^{\prime}\left(z_{0}\right)^{2}\right\}+O\left(\rho^{3}\right) \leq 0 .
$$

Since $\hat{p}^{\prime}(z)$ is analytic and not identically zero on $\widetilde{B}$, we may now invoke Schiffer's basic theorem of the method of boundary variation $[2, \S 10.3]$ to conclude from (5) that $\widetilde{B}$ is the union of analytic arcs lying on the trajectories of the quadratic differential $\hat{p}^{\prime}(z)^{2} d z^{2}<0$. (These are the trajectories orthogonal to those found by Duren and Schiffer [3] for the lower bound problem.) 
Parametrizing each component $\widetilde{B}_{i}$ of $\widetilde{B}$ by $z=z(s)$, we conclude that

$$
\frac{d}{d s} \widehat{p}(z(s))=\hat{p}^{\prime}(z(s)) z^{\prime}(s)
$$

is purely imaginary, or that

$$
\frac{\partial}{\partial s} \widehat{g}(z)=\frac{\partial}{\partial s} \operatorname{Re}\{\widehat{p}(z)\}=0
$$

on $\widetilde{B}$. In other words, $\widehat{g}(z)=c_{i}$ on $\widetilde{B}_{i}$ for $i=1, \ldots, m-k$ and some constants $c_{i}>0$.

The $c_{i}$ are now easily computed in terms of conformal invariants. This is a key computation, for it leads to an explicit expression for the maximal capacity. Let $\widetilde{\omega}_{i}(z)$ be the harmonic measure of $\widetilde{\Gamma}_{i}$ with respect to $\widetilde{\Omega}$. By definition, Green's function $\widehat{g}$ for $\widehat{\Omega}$ is identically zero on $\widetilde{A}$, and we have shown that $\widehat{g} \equiv c_{i}$ on $\widetilde{B}_{i}=\widetilde{\Gamma}_{i+k}$, for $i=1, \ldots, m-k$. Thus the function

$$
h(z)=\widehat{g}(z)-\sum_{i=1}^{m-k} c_{i} \widetilde{\omega}_{i+k}(z)
$$

is harmonic in the domain $\widetilde{\boldsymbol{\Omega}} \backslash\{\infty\}$, has a logarithmic singularity at infinity, and is identically zero on the entire boundary $\widetilde{\Gamma}$. We conclude that $h(z)$ is Green's function $\widetilde{g}$ of $\widetilde{\Omega}$ :

$$
\widetilde{g}(z)=\tilde{g}(z ; \infty)=\widehat{g}(z)-\sum_{i=1}^{m-k} c_{i} \widetilde{\omega}_{i+k}(z) .
$$

Now fix $j \in\{1, \ldots, m-k\}$. Since $\widetilde{B}_{j}$ is a union of analytic arcs, the normal direction is defined almost everywhere. Therefore, we may integrate the derivative with respect to the outer normal of both sides of (6) about the curve $\widetilde{B}_{j}$ :

$$
\int_{\widetilde{B}_{j}} \frac{\partial \widetilde{g}}{\partial n}|d z|=\int_{\widetilde{B}_{j}} \frac{\partial \widehat{g}}{\partial n}|d z|-\sum_{i=1}^{m-k} c_{i} \int_{\widetilde{B}_{j}} \frac{\partial \widetilde{\omega}_{i+k}}{\partial n}|d z| .
$$

We have

$$
\int_{\widetilde{B}_{j}} \frac{\partial \widetilde{\omega}_{i+k}}{\partial n}|d z|=2 \pi p_{i j}
$$

by the definition of $p_{i j}$. Also, since $\widehat{g}$ is harmonic in the finite domain bounded by $\widetilde{B}_{j}$, we have

$$
\int_{\widetilde{B}_{j}} \frac{\partial \widehat{g}}{\partial n}|d z|=0
$$

Finally,

$$
\int_{\widetilde{B}_{j}} \frac{\partial \widetilde{g}}{\partial n}|d z|=-2 \pi \widetilde{\omega}_{j+k}(\infty)=-2 \pi \omega_{j+k}(\infty)
$$


Thus, (7) reduces to the equation

$$
\omega_{j+k}(\infty)=\sum_{i=1}^{m-k} c_{i} p_{i j} .
$$

Repeating this calculation for each $j \in\{1, \ldots, m-k\}$, we find that $\mathbf{P} c=\mathbf{v}$. But $\mathbf{P}$ is invertible. To see this, recall the well-known argument that for the $m \times m$ Riemann matrix $\mathbf{R}=\left(r_{i j}\right)$ of an arbitrary $m$-connected domain $D$, the $(m-1) \times(m-1)$ submatrix $\mathbf{R}^{\prime}=\left(r_{i j}\right)$, for $i, j=1, \ldots, m-1$ is positive definite, hence invertible. (See, for instance, Nehari [7, Chapter I, §10].) The invertibility of $\mathbf{P}$ follows, since any square submatrix centered on the diagonal of a positive definite matrix is also positive definite.

Robin's constant for the domain $\widehat{\Omega}$ is computed directly from equation (6). The extremal capacity $d(\widetilde{A})$ is then seen to be the right-hand side of (3). But observe that none of the previous calculations depend on the extremal character of the domain. The fact that $\widehat{g}$ is constant on each $\widetilde{B}_{i}$ completely determines the $c_{i}$ and the capacity of the image of $A$, which is thus maximal. In other words, the extremal domains are characterized by the fact that $\hat{g}$ is constant on each $\widetilde{B}_{i}$. This completes the proof of the theorem .

\section{The GENeral CASE}

We now consider general subsets $A$ of $\Gamma$. Since an arbitrary admissible $f$ may not induce a one-to-one correspondence between boundary points, we first need to agree on a definition for the set $f(A)$. Let $C(f, \zeta)$ denote the cluster set of $f$ at the point $\zeta$. Then we define $f(A)=\{C(f, \zeta): \zeta \in A\}$. The somewhat surprising result is that the sharp upper bound for $d(f(A))$ is still given by Theorem 1. In other words, if a component of $\Gamma$ contains even a single point of $A$, then the maximum value of $d(f(A))$ is the same as if $A$ contained that full boundary component.

Theorem 2. Let $\Omega$ be a domain containing $\infty$ and bounded by the Jordan curves $\Gamma_{1}, \ldots, \Gamma_{m}$. For $k \leq m$, suppose $A \subset \bigcup_{i=1}^{k} \Gamma_{i}$ and $A \cap \Gamma_{i} \neq \varnothing$ for $i=$ $1, \ldots, k$. Let $[A]=\bigcup_{i=1}^{k} \Gamma_{i}$. Then

$$
\max _{f \in \mathscr{F}} d(f(A))=\max _{f \in \mathscr{F}} d(f([A])) .
$$

Note that the theorem includes the case where $A$ has points in each of the components of $\Gamma$ (that is, $k=m$ ). Then there is some $f \in \mathscr{F}$ attaining the trivial upper bound $d(f(A))=d(\Gamma)$.

Proof of theorem. Suppose first that $A \subset \Gamma_{1}$. For the sake of clarity, we carry out the proof under the stronger assumption that $A$ contains some subarc $I$ in $\Gamma_{1}$. We then indicate how to modify the argument to handle the general situation.

Without loss of generality, we may assume that $\Omega$ is already the extremal configuration maximizing $d(f([A]))=d\left(f\left(\Gamma_{1}\right)\right)$. In other words, $d([A]) \geq$ $d(f([A]))$ for all $f \in \mathscr{F}$. Let $\widehat{\Omega}$ be the domain containing $\Omega$ and bounded only by $[A]=\Gamma_{1}$. Note that any map in the family $\widehat{\mathscr{F}}$ of normalized conformal 
maps of $\widehat{\Omega}$ is also in $\mathscr{F}$, by restriction, and it preserves the maximal capacity $d([A])$. The idea is to find a function $F \in \widehat{\mathscr{F}}$ so that $F(A)=F([A])$. We will then have

$$
d(F(A))=d(F([A]))=d([A]),
$$

and the theorem will be proved.

But the construction of $F$ is fairly straightforward. The Riemann mapping theorem gives us a function $f_{1}$ mapping $\widehat{\Omega}$ to the complement of the unit disk D, with $f_{1}(\infty)=\infty$. Then $f_{1}(I)$ is a subarc of $f_{1}(A) \subset \partial \mathbf{D}$. Next, there is a Möbius transformation $f_{2}$ taking the arc $f_{1}(I)$ to the top half of the unit circle. (This will not preserve infinity.) After applying $f_{1}$ and $f_{2}$, we have reduced the problem to the example of the unit circle described in the introduction. If $f_{3}$ is the Joukowski map, then

$$
f_{3} \circ f_{2} \circ f_{1}([A])=f_{3} \circ f_{2} \circ f_{1}(A)=\{x:-2 \leq x \leq 2\} .
$$

But infinity has moved to a finite point, and the derivative at infinity is not 1 . We can achieve the correct normalization by following with a Möbius transformation $f_{4}$. The function $F=f_{4} \circ f_{3} \circ f_{2} \circ f_{1}$ is in $\widehat{\mathscr{F}}$ and $F([A])=F(A)$ is a circular arc.

If $f_{1}(I)$ is the arc from $e^{i \sigma}$ to $e^{i(\pi-\sigma)}$, where $0<\sigma<\frac{\pi}{2}$, the maps $f_{2}$ and $f_{4}$ are given explicitly by

$$
f_{2}(z)=\frac{z-i r}{1+i r z}, \quad f_{4}(z)=\frac{r^{2}}{1-r^{4}}\left(\frac{1}{z+i\left(\frac{1}{r}-r\right)}\right),
$$

where $r=\sqrt{\tan \left(\frac{\sigma}{2}\right)}$.

This proves the theorem when $A \subset \Gamma_{1}$ and $A$ contains an arc. Now suppose only that $A \subset \Gamma_{1}$. Kuester [6] has constructed a simply-connected domain $G$ containing infinity with the property that the normalized conformal map $F$ of $\widehat{\Omega}$ onto $G$ satisfies $C(F, \zeta)=\partial G$ for every $\zeta$ in $\Gamma$. In this case $F(A)=F([A])=\partial G$, by our definition of the boundary set $F(A)$, and again we have $d(F(A))=d([A])$.

Suppose next that $A \subset \Gamma_{1} \cup \cdots \cup \Gamma_{k}$, where $1<k \leq m$. Again, we may assume that $d([A]) \geq d(f([A]))$ for all $f \in \mathscr{F}$. The idea here is simply to iterate the process already described, component by component.

Let $\widehat{\Omega}_{1}$ denote the domain containing $\Omega$ and bounded only by $\Gamma_{1}$, and $\widehat{\Omega}$ that bounded by $[A]$. As we have already shown, there is a normalized map $F_{1}$ of $\widehat{\Omega}_{1}$ such that $F_{1}\left(A \cap \Gamma_{1}\right)=F_{1}\left(\Gamma_{1}\right)$. By restriction, $F_{1}$ belongs to $\mathscr{F}$ and $\widehat{\mathscr{F}}$, and so preserves the extremal capacity $d([A])$.

Now let $\widehat{\Omega}_{2}$ be the domain bounded only by $F_{1}\left(\Gamma_{2}\right)$. Again appealing to the construction given above, we know there is a normalized map $F_{2}$ of $\widehat{\Omega}_{2}$ so that

$$
F_{2}\left(F_{1}\left(A \cap \Gamma_{2}\right)\right)=F_{2}\left(F_{1}\left(\Gamma_{2}\right)\right) .
$$

The function $F_{2} \circ F_{1}$ belongs to $\mathscr{F}$ and $\widehat{\mathscr{F}}$, and since $F_{1}\left(\Gamma_{1}\right)$ is contained in $\widehat{\Omega}_{2}$, we still have

$$
F_{2}\left(F_{1}\left(A \cap \Gamma_{1}\right)\right)=F_{2}\left(F_{1}\left(\Gamma_{1}\right)\right)
$$


Repeating the above process for each of the remaining components, we get $k$ maps $F_{1}, \ldots, F_{k}$ belonging to $\mathscr{F}$ and $\widehat{\mathscr{F}}$ which satisfy

$$
F_{k} \circ F_{k-1} \circ \cdots \circ F_{1}\left(A \cap \Gamma_{i}\right)=F_{k} \circ F_{k-1} \circ \cdots \circ F_{1}\left(\Gamma_{i}\right),
$$

for $i=1, \ldots, k$. Thus, setting $F=F_{k} \circ \cdots \circ F_{1}$, we have $F(A)=F([A])$, and

$$
d(F(A))=d(F([A]))=d([A]) .
$$

This completes the proof.

\section{AN EXAMPLE}

Theorem 1 gives us a way of identifying an extremal domain in the case where $A$ is a single component of the boundary of the $m$-connected domain $\Omega$. It is well known that $\Omega$ can be mapped by a function $F \in \mathscr{F}$ to the exterior of a disk of some radius $R$, centered at the origin, with $m-1$ concentric circular slits, in such a way that $A$ is mapped to the boundary of the disk. (See Nehari [7, Chapter VII], for example.) But then $\widehat{g}(z)=\log (|z| / R)$ is Green's function for the domain $\widehat{\Omega}$ bounded only by $F(A)$, and is constant on each of the remaining boundary arcs. By Theorem 1, then, $d(F(A))$ is maximal. Intuitively, the set $A$ is spread out to increase its capacity, while the other components are forced to "hug" as close as conformally possible to $A$ to maintain the capacity of the full boundary. By contrast, an extremal configuration minimizing the capacity of $A$ is the exterior of a (smaller) circle with $m-1$ radial slits [3].

As an example, consider the ring domain $\Omega$ bounded by circles $B=\{\zeta$ : $|\zeta|=1\}$ and $A=\{\zeta:|\zeta-c|=r\}$, where $c$ is real and $c-r>1$. We use two methods to compute the maximal capacity $d(F(A))$ in terms of the defining parameters $c$ and $r$. First, we calculate the right-hand side of (3). And second, we explicitly construct the conformal map $F$ giving the extremal configuration described above. The capacity $d(F(A))$ is then just the radius of the circle $F(A)$. (See Figure 1.)

We begin both methods by mapping $\Omega$ to an annulus. The values $s$ and $t$ solving the system

$$
s t=1, \quad(c-s)(c-t)=r^{2}
$$

are the points on the positive real axis which are symmetric to one another with respect to both circles $A$ and $B$. If we choose $s<t$, the Möbius transforma-

$\Omega$

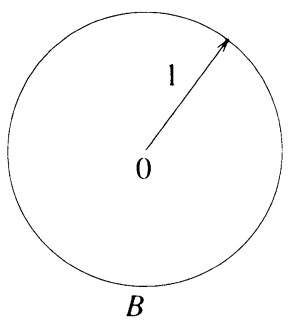

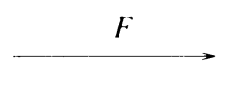

A
$F(\Omega)$



FIGURE 1. $d(F(A)$ is maximal. 
tion

$$
h(\zeta)=\tau \frac{z-s}{z-t}, \quad \tau=\frac{c+r-t}{c+r-s}
$$

maps $\Omega$ to an annulus $R$, centered at the origin, of outer radius 1 and inner radius $\mu=\frac{\tau}{t}$, with $A$ mapped to the unit circle, and $h(\infty)=\tau$.

Method 1. Observe that the harmonic measure of $B$ with respect to $\Omega$ is $\log |h(\zeta)| / \log \mu$. Thus, using the notation of Theorem $1, v_{1}=\omega_{2}(\infty)=$ $\log \tau / \log \mu$. A calculation shows that the period of the harmonic conjugate of $\omega_{2}$ about $B$ is $p_{11}=-(\log \mu)^{-1}$. Thus the maximal capacity is given by

$$
d(F(A))=d(\Gamma) e^{-c_{1} v_{1}}=d(\Gamma) \exp \left\{-\frac{v_{1}^{2}}{p_{11}}\right\}=d(\Gamma) \exp \left\{\frac{(\log \tau)^{2}}{\log \mu}\right\} .
$$

It remains to compute the capacity of the full boundary $\Gamma$. It is known that Green's function with singularity at $z=\tau$ for the annulus $R$ is given by

$$
g_{R}(z ; \tau)=\log \tau\left(\frac{\log |z|}{\log \mu}-1\right)-\log |p(z)|,
$$

where

$$
p(z)=\prod_{k=1}^{\infty} \frac{\left(1-\mu^{2 k-2} \frac{z}{\tau}\right)\left(1-\mu^{2 k} \frac{\tau}{z}\right)}{\left(1-\mu^{2 k-2} \tau z\right)\left(1-\mu^{2 k} \frac{1}{\tau z}\right)} .
$$

(See Henrici [5, pp. 259-263], for instance.) In view of the relations

$$
z \equiv e^{2 \pi i v}, \quad \mu \equiv e^{2 \pi i \tilde{\mu}}, \quad \tau \equiv e^{2 \pi i \tilde{\tau}},
$$

$p(z)$ can be expressed in terms of the standard elliptic theta function

$$
\theta_{4}(v)=\theta_{4}(v ; \mu)=\mu_{0} \prod_{k=1}^{\infty}\left(1-\mu^{2 k-1} e^{2 \pi i v}\right)\left(1-\mu^{2 k-1} e^{-2 \pi i v}\right),
$$

where $\mu_{0}=\prod_{k=1}^{\infty}\left(1-\mu^{2 k}\right)$, by

$$
p(z)=p^{*}(v(z)), \quad p^{*}(v)=\frac{\theta_{4}(v-(\tilde{\mu}+\tilde{\tau}))}{\theta_{4}(v-(\tilde{\mu}-\tilde{\tau}))} .
$$

A rather lengthy computation then shows that Robin's constant for $\Omega$ is

$$
\begin{aligned}
\gamma & =\lim _{\zeta \rightarrow \infty}\{g(\zeta ; \infty)-\log |\zeta|\} \\
& =\lim _{z \rightarrow \tau}\left\{g_{R}(z ; \tau)-\log \left|h^{-1}(z)\right|\right\} \\
& =\log \left(\frac{\mu}{\tau^{2}-\mu^{2}}\right)+\frac{(\log \tau)^{2}}{\log \mu}-\log \left|\frac{1}{2 \pi} \frac{\theta_{4}^{\prime}(-\widetilde{\mu})}{\theta_{4}(2 \tilde{\tau}-\widetilde{\mu})}\right|,
\end{aligned}
$$

so that

$$
d(\Gamma)=e^{-\gamma}=\frac{1}{2 \pi}\left(\frac{\tau^{2}}{\mu}-\mu\right) \exp \left\{-\frac{(\log \tau)^{2}}{\log \mu}\right\}\left|\frac{\theta_{4}^{\prime}(-\widetilde{\mu})}{\theta_{4}(2 \widetilde{\tau}-\widetilde{\mu})}\right| .
$$

The maximal capacity is thus

$$
d(F(A))=\frac{1}{2 \pi}\left(\frac{\tau^{2}}{\mu}-\mu\right)\left|\frac{\theta_{4}^{\prime}(-\widetilde{\mu})}{\theta_{4}(2 \widetilde{\tau}-\widetilde{\mu})}\right| .
$$


Method 2. Nehari shows via an exercise [7, p. 377, exercise 7] that the map $P(z)$ taking a finite multiply-connected domain $D$ with boundary $C$ to the interior of a disk centered at the origin with concentric slits must be given by

$$
\log (P(z))=\log \left(z-z_{0}\right)+\sum_{n=-\infty}^{\infty} a_{n} u_{n}(z),
$$

where $z_{0}$ is the pre-image of zero in $D$, the sequence $\left\{u_{n}(z)\right\}$ is a complete orthonormal set in $D$ with respect to the inner product

$$
(f, g)=\iint_{D} f^{\prime}(z) \overline{g^{\prime}(z)} d x d y,
$$

and the Fourier coefficients $a_{n}$ are given by

$$
a_{n}=\left(\log \frac{P(z)}{z-z_{0}}, u_{n}(z)\right)=\pi \overline{u_{n}\left(z_{0}\right)}-\frac{1}{2 i} \int_{C} \frac{\overline{u_{n}(z)}}{z-z_{0}} d z .
$$

The outer component of $C$ is mapped to the full circle, while each of the inner components is mapped to a slit. $P$ is uniquely determined by prescribing the values $z_{0}$ and $P^{\prime}\left(z_{0}\right)$.

In our case, $\left\{u_{n}\right\}$ and $\left\{a_{n}\right\}$ can be calculated explicitly when $D$ is taken to be the annulus $R=h(\Omega)$ and $z_{0}$ is $\tau$. The collection $\left\{z^{n}\right\}$, where $n$ ranges over all integers, is complete and orthogonal with respect to the inner product, so we may take the orthonormal set $\left\{u_{n}\right\}$ to be

$$
u_{n}(z)=\frac{z^{n}}{\sqrt{\pi n\left(1-\mu^{2 n}\right)}} .
$$

The $a_{n}$ are then calculated to be

$$
a_{n}= \begin{cases}\sqrt{\frac{\pi}{n\left(1-\mu^{2 n}\right)}}\left(\tau^{n}-\tau^{-n}\right), & n=-1,-2, \ldots, \\ \sqrt{\frac{\pi}{n\left(1-\mu^{2 n}\right)}}\left(\tau^{n}-\mu^{2 n} \tau^{-n}\right), & n=1,2, \ldots\end{cases}
$$

Thus, if we write

$$
S(z)=\sum_{n=-1}^{-\infty} \frac{\tau^{n}-\tau^{-n}}{1-\mu^{2 n}} \frac{z^{n}}{n}+\sum_{n=1}^{\infty} \frac{\tau^{n}-\mu^{2 n} \tau^{-n}}{1-\mu^{2 n}} \frac{z^{n}}{n},
$$

then $P(z)=(z-\tau) e^{S(z)}$.

If we follow $P(h(\zeta))$ by an inversion about zero, the result will map $\Omega$ to the desired extremal configuration, with infintiy preserved. The normalized extremal map is then calculated to be

$$
F(\zeta)=\left(\frac{\tau^{2}}{\mu}-\mu\right) \frac{e^{S(\tau)}}{P(h(\zeta))} .
$$

To compute the radius of the circle $F(A)$, and hence the the maximal capacity, recall that the outer boundary $h(A)$ of $R$ is the unit circle. Since $P(1)$ 
TABLE 1. Extremal configurations (See Figure 1.)

\begin{tabular}{|c|c|c|c|c|}
\hline$c$ & $r=d(A)$ & $d(\Gamma)$ & $R_{A}=d(F(A))$ & $R_{B}$ \\
\hline 2.01 & 1 & 1.573 & 1.497 & 1.869 \\
2.1 & 1 & 1.597 & 1.364 & 1.885 \\
2.5 & 1 & 1.700 & 1.202 & 2.404 \\
3 & 1 & 1.825 & 1.128 & 2.952 \\
4 & 1 & 2.061 & 1.067 & 3.998 \\
10 & 1 & 3.179 & 1.010 & 9.999 \\
4 & 2 & 2.658 & 2.138 & 3.908 \\
5 & 3 & 3.549 & 3.130 & 4.878 \\
12 & 10 & 10.255 & 10.074 & 11.696 \\
3 & .5 & 1.559 & .563 & 2.989 \\
3 & .1 & 1.318 & .113 & 3.000 \\
3 & .01 & 1.198 & .0113 & 3.000 \\
\hline
\end{tabular}

is real and positive, it is easily seen that

$$
d(F(A))=F\left(h^{-1}(1)\right)=\frac{\left(\tau^{2} / \mu-\mu\right)}{(1-\tau)} e^{S(\tau)-S(1)} .
$$

It is possible to reduce equation (8) to equation (10) using known identities for theta functions (see the Bateman Manuscript Project [1, §13.19], for example). Introducing

$$
\theta_{1}(v)=\theta_{1}(v ; \mu)=2 \mu_{0} \mu^{\frac{1}{4}} \sin \pi v \prod_{k=1}^{\infty}\left(1-\mu^{2 k} e^{2 \pi i v}\right)\left(1-\mu^{2 k} e^{-2 \pi i v}\right),
$$

and using the fact that $\theta_{1}$ is odd, we can apply the identity $\theta_{4}(v+\widetilde{\mu})=$ $i B(v) \theta_{1}(v)$, where $B(v)=\exp \left(-i \pi\left(v+\frac{1}{2} \widetilde{\mu}\right)\right)$ to write

$$
\frac{\theta_{4}^{\prime}(-\widetilde{\mu})}{\theta_{4}(2 \widetilde{\tau}-\widetilde{\mu})}=\frac{1}{\tau} \frac{\theta_{1}^{\prime}(0)}{\theta_{1}(2 \widetilde{\tau})} \text {. }
$$

Then we can use the identity (corrected from [1, p. 358])

$$
\log \left[\pi \frac{\theta_{1}(v)}{\theta_{1}^{\prime}(0)}\right]=\log (\sin \pi v)+4 \sum_{n=1}^{\infty} \frac{\mu^{2 n}}{1-\mu^{2 n}} \frac{\sin ^{2} n \pi v}{n}
$$

to express the logarithm of equation (8) as an infinite series equivalent to the logarithm of equation (10).

The extremal maps (9), corresponding to various values of $c$ and $r$, were calculated and plotted using Mathematica. The results are summarized in Table 1.

\section{ACKNOWLEDGMENT}

The results of this paper form a portion of my Ph.D. dissertation. I would like to thank Peter Duren, my adviser, for many helpful conversations, and for his thoughtful editing. 


\section{REFERENCES}

1. Bateman Manuscript Project (A. Erdélyi, W. Magnus, F. Oberhettinger, and F. Tricomi, ed.), Higher transcendental functions, Vol. II, McGraw-Hill, New York, 1953.

2. P. Duren, Univalent functions, Springer-Verlag, New York, 1983.

3. P. Duren and M. M. Schiffer, Robin functions and distortion of capacity under conformal mapping, Complex Variables Theory Appl. 21 (1993), 189-196.

4. G. M. Goluzin, Geometric theory of functions of a complex variable, Transl. Math. Monos., Vol. 26, Amer. Math. Soc., Providence, RI, 1969.

5. P. Henrici, Applied and computational complex analysis, Vol. 3, Wiley, New York, 1986.

6. J. Kuester, A region whose prime ends all have the same impression, Math. Z. 136 (1974), $1-5$.

7. Z. Nehari, Conformal mapping, McGraw-Hill, New York, 1952.

8. M. M. Schiffer, Hadamard's forumla and variation of domain-functions, Amer. J. Math. 68 (1946), 417-448.

9. G. C. Wen, Conformal mappings and boundary value problems, Transl. Math. Monos., Vol. 106, Amer. Math. Soc., Providence, RI, 1992.

Department of Mathematics, University of Michigan, Ann Arbor, Michigan 48109

E-mail address: Robert_Thurman@um.cc.umich.edu 